\title{
Design of a Prospective Study on Pharmacokinetic-Guided Dosing of Prophylactic Factor Replacement in Hemophilia A and B (OPTI-CLOT TARGET Study)
}

Tine M.H.J. Goedhart ${ }^{1, *(1)} \quad$ Laura H. Bukkems ${ }^{2, *(-)}$ Michiel Coppens ${ }^{3}$ Karin J. Fijnvandraat ${ }^{4}$ Saskia E.M. Schols ${ }^{5}$ Roger E.G. Schutgens ${ }^{60}$ jeroen Eikenboom ${ }^{7}$ Floor C.J.I. Heubel-Moenen ${ }^{8}$ Paula F. Ypma $^{9} \quad$ L. Nieuwenhuizen ${ }^{10} \quad$ K. Meijer $^{11}$ Frank W. G. Leebeek ${ }^{12}$ Ron A.A. Mathôt ${ }^{2, *}$ Marjon H. Cnossen ${ }^{1, *}$

\footnotetext{
1 Department of Pediatric Hematology and Oncology, Erasmus MC Sophia Children's Hospital, University Medical Center Rotterdam, Rotterdam, The Netherlands

2 Department of Clinical Pharmacology - Hospital Pharmacy, Amsterdam UMC, University of Amsterdam, Amsterdam, The Netherlands

${ }^{3}$ Department of Vascular Medicine, Amsterdam Cardiovascular Sciences, Amsterdam UMC, University of Amsterdam, Amsterdam, The Netherlands

${ }^{4}$ Department of Pediatric Hematology, Amsterdam UMC, Emma Children's Hospital, University of Amsterdam, Meibergdreef 9, Amsterdam, the Netherlands

${ }^{5}$ Department of Hematology, Radboud University Medical Center, Nijmegen, and the Hemophilia Treatment Center NijmegenEindhoven-Maastricht, The Netherlands

6 Van Creveldkliniek, University Medical Center Utrecht, Utrecht, The Netherlands

7 Department of Internal Medicine, Division of Thrombosis and Hemostasis, Leiden University Medical Center, Leiden, The Netherlands
}

TH Open 2022;6:e60-e69
Address for correspondence Marjon $\mathrm{H}$. Cnossen, MD, PhD, Department of Pediatric Hematology and Oncology, Erasmus MC Sophia Children's Hospital, University Medical Center Rotterdam, Post office Box 2040, 3000 CA Rotterdam, The Netherlands (e-mail: m.cnossen@erasmusmc.nl).

\footnotetext{
${ }^{8}$ Department of Hematology, Maastricht University Medical Center, Maastricht, The Netherlands

${ }^{9}$ Department of Hematology, Haga Hospital, The Hague, The Netherlands

${ }^{10}$ Department of Internal Medicine, Maxima Medical Center, Veldhoven, The Netherlands

${ }^{11}$ Department of Hematology, University Medical Center Groningen, Groningen, The Netherlands

${ }^{12}$ Department of Hematology, Erasmus MC, University Medical Center Rotterdam, Rotterdam, The Netherlands
}
Abstract
In resource-rich countries, almost all severe hemophilia patients receive prophylactic replacement therapy with factor concentrates to prevent spontaneous bleeding in joints and muscles to decrease the development of arthropathy and risk of long-term

\section{Keywords}
- hemophilia
- pharmacokinetics
- factor VIII
- factor IX
- prophylaxis disability. Pharmacokinetic (PK)-guided dosing can be applied to individualize factor replacement therapy, as interindividual differences in PK parameters influence factor VIII (FVIII) and FIX activity levels. PK-guided dosing may therefore lead to more optimal safeguarding of FVIII/FIX levels during prophylaxis and on demand treatment. The OPTI-CLOT TARGET study is a multicenter, nonrandomized, prospective cohort study that aims to investigate the reliability and feasibility of PK-guided prophylactic dosing

\footnotetext{
Shared first and last authorships.
}

received

August 12, 2021 accepted after revision January 11, 2022
DOI https://doi.org/ 10.1055/a-1760-0105. ISSN 2512-9465.

\section{(c) 2022. The Author(s).}

This is an open access article published by Thieme under the terms of the Creative Commons Attribution License, permitting unrestricted use, distribution, and reproduction so long as the original work is properly cited. (https://creativecommons.org/licenses/by/4.0/)

Georg Thieme Verlag KG, Rüdigerstraße 14, 70469 Stuttgart, Germany 
of factor concentrates in hemophilia-A and -B patients in daily clinical practice. At least 50 patients of all ages on prophylactic treatment using standard half-life (SHL) and extended half-life (EHL) factor concentrates will be included during 9 months and will receive PK-guided treatment. As primary endpoint, a minimum of four FVIII/FIX levels will be compared with FVIII/FIX levels as predicted by Bayesian forecasting. Secondary endpoints are the association of FVIII and FIX levels with bleeding episodes and physical activity, expectations and experiences, economic analyses, and optimization of population PK models. This study will lead to more insight in the reliability and feasibility of PK-guided dosing in hemophilia patients. Moreover, it will contribute to personalization of treatment by greater knowledge of dosing regimens needed to prevent and treat bleeding in the individual patient and provide evidence to more clearly associate factor activity levels with bleeding risk.

\section{Introduction}

Hemophilia A and hemophilia $\mathrm{B}$ are $\mathrm{X}$-linked recessive bleeding disorders caused by a deficiency or dysfunction of coagulation factor VIII (FVIII) or FIX, respectively. Severe patients (FVIII/FIX $<0.01 \mathrm{IU} / \mathrm{mL}$ ) and some moderate-tosevere patients (FVIII/FIX: 0.01-0.05 IU/mL) suffer from spontaneous bleeding or bleeding after minimal trauma. Prophylactic treatment by intravenous administration of factor concentrates aims to prevent (spontaneous) bleedings in joint and muscles and subsequent arthropathy with potential long-term disability. ${ }^{1,2}$

During prophylaxis theoretically, FVIII/FIX trough levels is targeted to $>0.01 \mathrm{IU} / \mathrm{mL}$. This principle is based on observations by Ahlberg as early as in 1965 that bleeding phenotype and joint status are strikingly different between severe and moderate-to-severe hemophilia patients with only minimal baseline FVIII level differences ( $<0.01$ vs. $0.01-0.05 \mathrm{IU} / \mathrm{mL}$ ). ${ }^{3}$ To achieve these FVIII/FIX trough levels during prophylaxis, FVIII/FIX concentrates are mostly prescribed according to body weight. ${ }^{2}$ Remarkably, it is still not usual clinical practice to standardly measure and monitor trough FVIII/FIX levels when no bleeding occurs. To personalize dosing, information on trough FVIII/FIX levels is of value to establish if prophylaxis is adequate for each individual patient, during followup, and in varying circumstances and when dosing on demand. In addition, the lack of knowledge of achieved FVIII/FIX levels impedes proper switching to novel longacting factor concentrates due to uncertainties which trough FVIII/FIX target levels should be targeted to prolongate earlier effective prophylactic treatment to prevent bleeding, especially in relation to physical activity or sports.

\section{Pharmacokinetic-Guided Dosing}

Large interindividual variability exists in the pharmacokinetics (PKs) of FVIII/FIX concentrates as demonstrated by Björkman et al among others. ${ }^{4-6}$ To understand and predict the consequences of the interindividual variability of factor concentrates in individuals, population PK models have been constructed for prophylaxis $s^{4,7-32}$ and perioperative treatment $^{33}$ with standard half-life (SHL) and extended half-life (EHL) FVIII and FIX concentrates for hemophilia-A and -B patients, respectively. With these population PK models, Bayesian forecasting can be performed. Herewith, individual PK parameters are estimated which are subsequently used to calculate the adequate dose for an individual patient to achieve FVIII/FIX target levels, both trough and peak. The availability of population PK models has made limited sampling possible, making prior frequent blood sampling ( $>10$ blood samples) ${ }^{34}$ and a wash-out period redundant. PK-guided dosing has also been reported to not only be able to predict dosing requirements to attain certain target FVIII/FIX levels but also to decrease the amount of factor concentrates with concomitant reduction of costs. ${ }^{33}$ Carlsson et al was the first to report a dose and cost reduction of $30 \%$ of FVIII concentrate without an increase in bleeding events, when PK-guided prophylactic dosing was compared with standard prophylactic dosing in a small patient sample. ${ }^{35}$ However, a recent randomized controlled perioperative trial was not able to show a decrease in FVIII concentrate consumption, although achievement of FVIII target ranges was clearly more optimal. ${ }^{36}$

We hypothesize PK-guided dosing leads to individualization of prophylaxis which is in accordance with the recommendations of the subcommittee on FVIII, FIX, and rare bleeding disorders of the International Society on Thrombosis and Haemostasis (ISTH). ${ }^{37}$ PK-guided dosing may help achieve higher trough levels more efficiently when clinically indicated, as well as provide guidance when patients switch to alternative replacement factor concentrates, while taking cost and benefit of treatment into account. In addition, PK-guided dosing may lead to increased insight into the association between FVIII/FIX levels, bleeding (risk), and physical activity levels in individual patients as factor levels can be predicted at any time point and related to bleeding and activity. Therefore, we aim to prove that FVIII/FIX trough and peak levels as set by treating physician can be predicted and achieved reliably by application of PK-guided prophylaxis and that this intervention is feasible for patients and treatment teams.

\section{Objective}

Investigate the reliability and feasibility of PK-guided prophylactic dosing of factor concentrates in hemophilia-A and -B patients in daily clinical practice. 


\section{Methods}

\section{Study Design}

The OPTICLOT TARGET study is a multicenter, nonrandomized, prospective cohort study.

\section{Study Population}

Patients will be recruited from the two Dutch Hemophilia treatment Centers; follows Erasmus MC, University Medical Center Rotterdam and Amsterdam University Medical Centers.

\section{Inclusion Criteria}

Inclusion criteria are as follows:

- Hemophilia-A and -B patients of all ages on prophylaxis.

- Prophylaxis with SHL or EHL factor concentrates.

- Written (parental) informed consent, according to local law and regulations.

\section{Exclusion Criteria}

Exclusion criteria are listed below:

- Patients with other severe congenital or acquired hemostatic abnormalities.

- General medical conditions which may interfere with participation in the study.

- Inability to adhere to prophylaxis and/or inability to keep detailed logs on infusion and bleeding episodes.

- Withdrawal of (parental) informed consent.

- Presence of FVIII/FIX inhibitor, leading to alternative treatment with bypassing products, immune toleration induction, and/or other immune modulating treatment.

\section{Outcome Measures}

\section{Primary Endpoints}

Observed FVIII and FIX levels in comparison to FVIII and FIX levels are predicted by Bayesian forecasting. The predictive performance is deemed acceptable when at least $80 \%$ of the actual FVIII/FIX levels are within $\pm 25 \%$ of the predicted (target) values as stated by treating professional.

\section{Secondary Endpoints}

The four secondary endpoints are briefed below:

1. Association of (real world or predicted) FVIII/FIX levels with bleeding episodes and daily activities. Additionally, bleeds will be categorized according to the following subclassifications: total number of bleeds over time, number of spontaneous bleeds, number of traumatic bleeds, number of joint bleeds, number of target joint bleeds, and bleed severity.

2. Expectations, feasibility, and experience with PK-guided dosing with the different factor concentrates (SHL vs. EHL) as reported by patient/caretakers and physician will be measured using a visual analogue scale (VAS) questionnaire at the start and end of the study.
3. Economic analysis in which costs and benefits of standard prophylactic treatment and PK-guided prophylaxis are compared.

4. Analysis of described modifiers effecting PK parameters of FVIII/FIX concentrate to further optimize population PK models. Modifiers include demographics (such as lean body mass) and laboratory measurements (such as the von Willebrand Factor levels).

\section{Interventions}

Study interventions are depicted in the flowchart ( $\mathbf{- F i g . 1}$ ). Patients will be categorized into strata according to type of hemophilia and type of factor concentrate (SHL or EHL). For all patients, the following patient characteristics and demographics will be collected: type of hemophilia, endogenous factor level, DNA mutation, age, height $(\mathrm{cm})$, weight $(\mathrm{kg})$, body mass index (BMI; $\mathrm{kg} / \mathrm{cm}^{2}$ ), lean body mass $(\mathrm{kg})$, blood group, current other medication, and activity patterns.

Patients/caretakers will fill in the Hemophilia Activities List (HAL) and/or pediatric HAL (PedHAL) before initiation. Moreover, the Hemophilia Joint Health Score (HJHS) will be performed or must have been performed $<12$ months prior inclusion.

The validated PedHAL/HAL questionnaire and the HJHS are included as clinical parameters to systematically establish baseline values of functional outcome from the patient's perspective and to be informed of joint status, respectively. These clinical parameters may help to evaluate outcomes after implementation of PK guidance.

Furthermore, both patients/caretakers and the treating physician will fill in a specifically developed questionnaire using VAS scales, before the implementation of PK-guided dosing, considering the expectations with PK-guided dosing of prophylaxis. More specifically, in the questionnaire, questions are asked about satisfaction, being informed of factor levels, and expected burden of PK guidance. Moreover, when patients switch to an EHL factor concentrate, the reasons for switching are also asked.

An individual PK profile will be constructed after a factor concentrate dose of 35 to $50 \mathrm{IU} / \mathrm{kg}$, depending on hemophilia type and age of the patient. The frequency and timing of blood sampling during PK profiling is depending on type of hemophilia and type of factor concentrate (-Fig. 2). No wash out period is required if three prior infusions and time points of infusion are documented. During sampling of the PK profile, laboratory tests will be performed according to - Tables 1 and 2.

Dosing will be advised by clinical pharmacologist on the basis of FVIII/FIX target trough levels as set by treating physician, in accordance to patient characteristics, previous trough levels (if a patient switches between factor concentrates), bleeding history, activity pattern, and in consultation with patient/caretakers. If desirable, physicians are also able to set FVIII/FIX target peak levels during intensive physical activities. In this way, treatment is truly customized and tailored to the needs and lifestyle of each individual as personalization is meant to be. Retrospective data of a patient, such as previous trough levels and factor levels at 


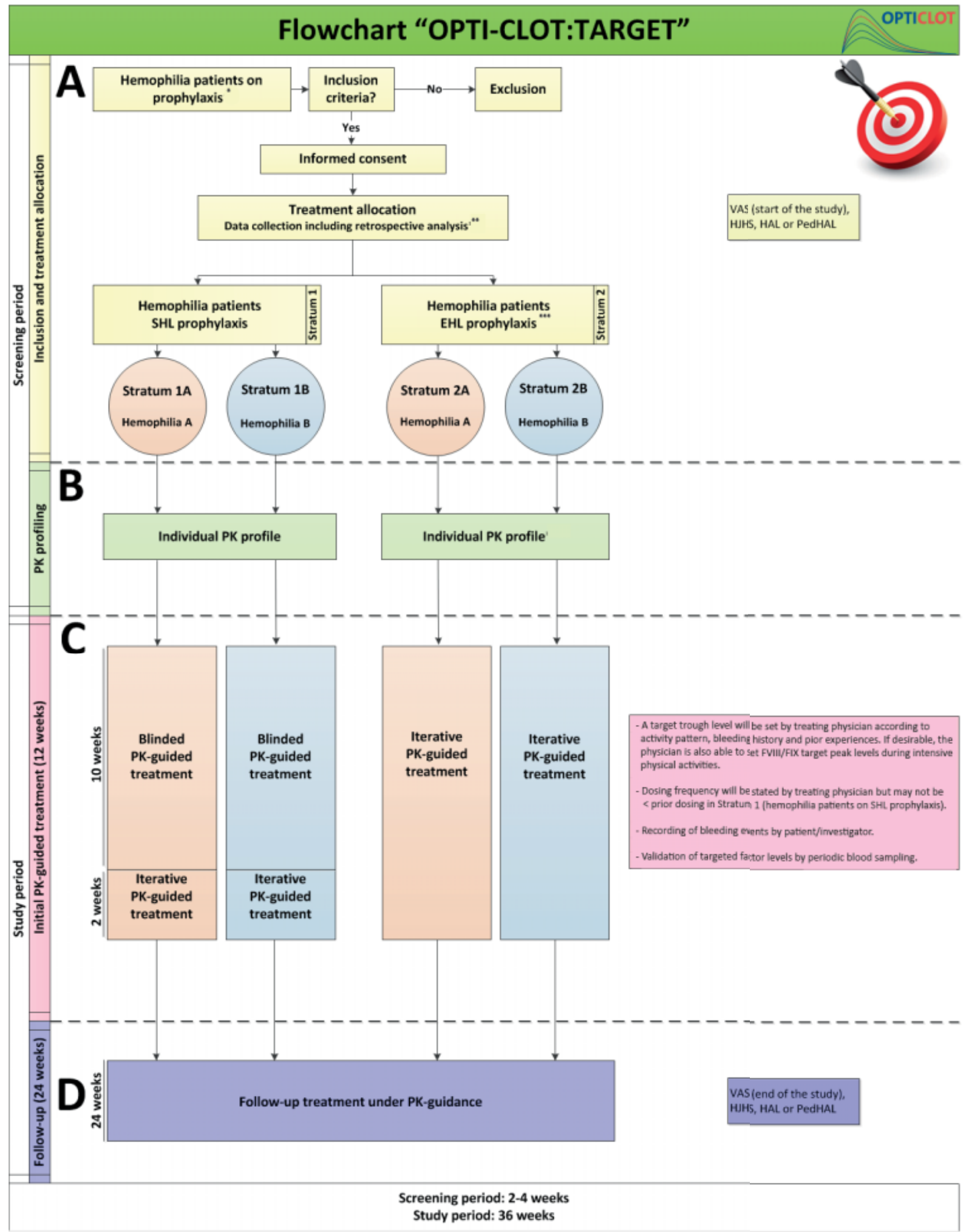

Fig. 1 Flow chart. EHL, extended half-life; HAL, Hemophilia Activity List; HJHS, Hemophilia Joint Score; PedHAL, pediatric HAL; PK, pharmacokinetic(s); SHL, standard half-life; VAS, visual analogue scale *Non severe hemophilia patients will be analyzed separately. ${ }^{* *}$ In parallel with the prospective study, retrospective data analysis will be performed over a 12 month period prior to inclusion (if no PK profiling has been performed) or from PK profiling prior to inclusion. ${ }^{* *}$ Patients in stratum 2 could undergo PK profiling during SHL prophylaxis as well as during EHL prophylaxis. 


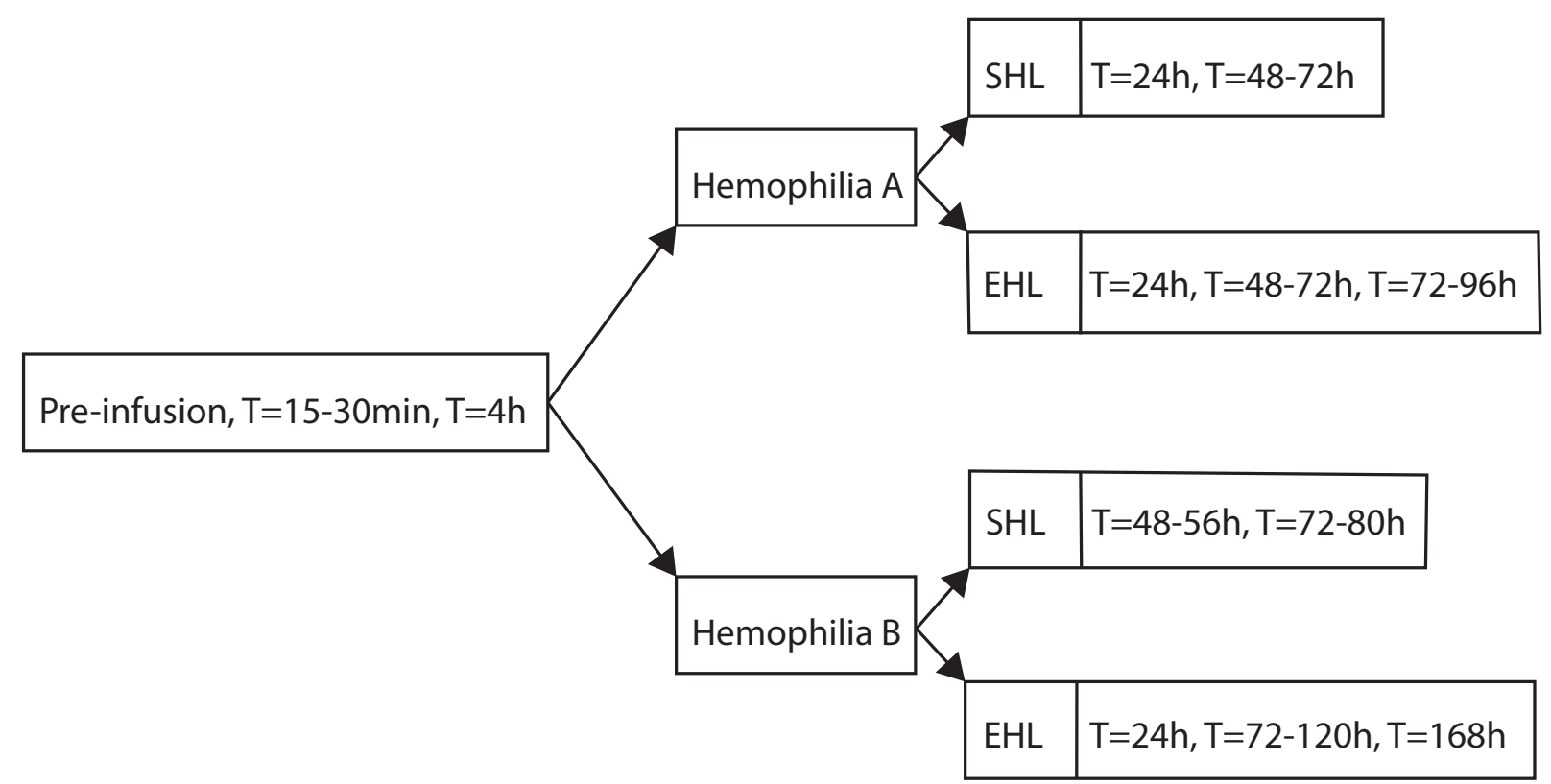

Fig. 2 Time points (T) of laboratory tests during individual PK-profiling. A preinfusion, $t=15-30$ minutes and $\mathrm{t}=4$ hours sample (left) are performed in all patients. The other time points (right) depend on hemophilia type and brand of factor concentrate. EHL, extended half-life; PK, pharmacokinetic; SHL, standard half-life.

onset of a bleed or during sport activities, can be informative to the physician to set target levels.

Thereafter, patients will initially be on PK-guided treatment for 12 weeks. During these 12 weeks, a minimum of three factor levels will be measured and compared with predicted FVIII/FIX values to validate predicted dosing regimen (-Table 3). Patients on EHL will be on iterative PKguided treatment with dose adjustment if needed based on both factor levels and bleedings. Iterative treatment is desirable in these patients as most patients initiate treatment with EHL factor concentrates after being on prophylaxis with SHL factor concentrates. Because of the lack of knowledge of most optimal (frequency and dose of) EHL factor concentrate, this period has a dose finding perspective.

For patients on SHL predicted FVIII/FIX values will be blinded to the treating physician and dosages will not be adjusted during the first 10 weeks. Thereafter, dose adjustment can be made.

A subsequent follow-up period of 24 weeks on PK-guided treatment is necessary to further collect data to establish the associations between FVIII/FIX levels and bleeding events. Only if clinically indicated, FVIII/FIX levels will be measured during bleeds ( - Table 3 ). At the end of this follow-up period, one final blood sample will be taken to compare the factor level with the predicted value (-Table 3). Patients/caretakers will again fill in the HAL or PedHAL questionnaire and the physiotherapist will perform the HJHS to evaluate outcomes after implementation of PK-guidance.

Finally, at the end of the study, both patients/caretakers and the treating physician will fill in the VAS questionnaire, considering the experience with PK-guided dosing and EHL factor concentrate when patients have switched to an EHL factor concentrate.
Importantly, hemophilia patients, who have undergone individual PK-profiling prior to study inclusion or who have already received PK-guided treatment on SHL or EHL concentrate, are also able to participate in the study. PK profiling is required to be performed with a maximum of 1 year prior to study inclusion when $<12$ years of age and a maximum of 3 years prior to study inclusion when 12 years and older. Patients who already received PK guidance prior to study inclusion, will only complete the VAS questionnaire at the end of the study period, since asking the patient questions with regard to expectations on PK-guided dosing at the beginning of the study would lead to recall bias. Also, the HJHS will not be performed in these patients and PedHAL will not be completed in this subgroup as results after body weight (and bleeding) -based prophylaxis and PK-guided therapy cannot be compared.

In parallel with the prospective study, retrospective data analysis will be performed over a 12-month period prior to inclusion (if no PK profiling has been performed) or from PK profiling prior to inclusion. These data will be utilized as "real-world data" to construct and enrich available population PK models. Moreover, if patients kept a detailed patient log-on infusion dates and timing and bleeding episodes, annualized (joint) bleeding rate (A(J)BR) FVIII/FIX trough levels and FVIII/FIX levels during physical activities and at the onset of a bleed can be calculated.

Factor activity levels will be measured by local laboratories, as this reflects the real-world setting of standard clinical practice. Plasma samples are stored in case centralized measurements are deemed necessary. Laboratory specifications (assay, reagents, deficient plasma, and analyzer) applied in local laboratory will be recorded precisely. 
Table 1 Laboratory tests during individual PK profiling for patients with hemophilia A

\begin{tabular}{|c|c|c|c|c|c|c|}
\hline On FVIII-SHL & Preinfusion & $\mathrm{T}=15-30$ minute & $\mathrm{T}=4$ hours & $\mathrm{T}=24$ hours & $\mathrm{T}=48-72$ hours & \\
\hline On FVIII-EHL & Preinfusion & $\mathrm{T}=15-30$ minute & $\mathrm{T}=4$ hours & $\mathrm{T}=24$ hours & $\mathrm{T}=48$ hours & $\mathrm{T}=72-96$ hours $^{\mathrm{a}}$ \\
\hline ASAT & $x$ & & & & & \\
\hline ALAT & $x$ & & & & & \\
\hline GGT & $x$ & & & & & \\
\hline $\mathrm{LDH}$ & $x$ & & & & & \\
\hline $\mathrm{AF}$ & $x$ & & & & & \\
\hline Albumin & $x$ & & & & & \\
\hline Urea & $x$ & & & & & \\
\hline Creatinine & $x$ & & & & & \\
\hline Hemoglobin & $x$ & & & & & \\
\hline Hematocrit & $x$ & & & & & \\
\hline Thrombocytes & $x$ & & & & & \\
\hline $\begin{array}{l}\text { Blood group } \\
\text { (if unknown) }\end{array}$ & $x$ & & & & & \\
\hline Factor VIII & $x$ & $x$ & $x$ & $x$ & $x$ & $x$ \\
\hline VWF:Ag & $x$ & $x$ & $x$ & $x$ & $x$ & $x$ \\
\hline VWF:Act & $x$ & $x$ & $x$ & $x$ & $x$ & $x$ \\
\hline VWF:CB & $x$ & $\mathrm{x}$ & $x$ & $x$ & $x$ & $x$ \\
\hline VWFpp & $x$ & $x$ & $x$ & $x$ & $x$ & $x$ \\
\hline $\begin{array}{l}\text { Inhibitor FVIII } \\
\text { (only } \geq 18 \text { years) }\end{array}$ & $x$ & & & & & \\
\hline Bethesda FVIII & $x$ & & & & & \\
\hline Buffycoat & $x$ & & & & & \\
\hline APTT & $x$ & $x$ & $x$ & $x$ & $x$ & $x$ \\
\hline $\mathrm{PT} / \mathrm{INR}$ & $x$ & & & & & \\
\hline Factor V & $x$ & & & & & \\
\hline Fibrinogen & $x$ & & & & & \\
\hline $\begin{array}{l}\text { Max of } 10-\mathrm{mL} \\
\text { citrate plasma }\end{array}$ & $\bar{x}$ & $x$ & $x$ & $x$ & $x$ & $x$ \\
\hline
\end{tabular}

Abbreviations: AF, alkaline phosphatase; ALAT, alanine aminotransaminase; APTT, activated partial thromboplastin time; ASAT, aspartate aminotransferase; EHL, extended half-life; FIX, factor IX; FV, factor V; FVIII, factor VIII; GGT, gamma-glutamyltransferase; INR, international normalized ratio; LDH, lactate dehydrogenase; PFA, platelet function assay; PK, pharmacokinetic; PT, prothrombin time; SHL, standard half-life; T, time point; VWF:Act, von Willebrand's factor activity; VWF:Ag, von Willebrand factor antigen; VWF:CB, von Willebrand factor collagen binding; VWFpp, von Willebrand factor propeptide.

${ }^{\mathrm{a}}$ Only in case of an EHL concentrate.

Preferably, the local laboratory assays match with the assays as used during population PK model construction.

\section{Bayesian Forecasting}

Bayesian forecasting will be performed with the NONMEM software (Icon, Dublin, Ireland); individual PK parameters will be assessed with a limited number of blood samples. Available population PK models in literature and new models that will become available will be used. Based on the estimated individual PK parameters and the FVIII/FIX target trough and peak values as set by the physician, dosing schedules will be calculated.

\section{Sample Size Calculation}

In this prospective study, we aim to evaluate the predictive performance of PK-guided dosing in hemophilia patients. It is not common practice to calculate a sample size for prognostic models, and, to the best of our knowledge, it is not possible to calculate a sample size for the determination of predictive performance. What we do know is that as characteristics, such as age, body weight, activity pattern, and bleeding phenotype, are not part of the inclusion or exclusion criteria, the study population will be a reflection of the real-world and thus a heterogeneous hemophilia population. However, we aim to enroll a minimum of 50 patients in all strata 
Table 2 Laboratory tests during individual PK profiling for patients with hemophilia B

\begin{tabular}{|c|c|c|c|c|c|c|}
\hline On FIX-SHL & Preinfusion & $\mathrm{T}=15-30$ minutes & $\mathrm{T}=4$ hours & $\mathrm{T}=48-56$ hours & $T=72-80$ hours & \\
\hline On FIX-EHL & Preinfusion & $\mathrm{T}=15-30$ minutes & $T=4$ hours & $\mathrm{T}=24$ hours & $\mathrm{T}=72-120$ hours & $T=168$ hours $^{a}$ \\
\hline ASAT & $x$ & & & & & \\
\hline ALAT & $x$ & & & & & \\
\hline GGT & $x$ & & & & & \\
\hline $\mathrm{LDH}$ & $x$ & & & & & \\
\hline $\mathrm{AF}$ & $x$ & & & & & \\
\hline Albumin & $x$ & & & & & \\
\hline Urea & $x$ & & & & & \\
\hline Creatinine & $x$ & & & & & \\
\hline Hemoglobin & $x$ & & & & & \\
\hline Hematocrit & $x$ & & & & & \\
\hline Thrombocytes & $x$ & & & & & \\
\hline $\begin{array}{l}\text { Blood group } \\
\text { (if unknown) }\end{array}$ & $x$ & & & & & \\
\hline Factor IX & $x$ & $x$ & $x$ & $x$ & $x$ & $x$ \\
\hline $\begin{array}{l}\text { Inhibitor FIX } \\
\text { (only } \geq 18 \text { years) }\end{array}$ & $x$ & & & & & \\
\hline Bethesda FIX & $x$ & & & & & \\
\hline Factor VIII & $x$ & & & & & \\
\hline VWF:Ag & $x$ & & & & & \\
\hline VWF:Act & $x$ & & & & & \\
\hline VWF:CB & $x$ & & & & & \\
\hline VWFpp & $x$ & & & & & \\
\hline Buffy coat & $\bar{x}$ & & & & & \\
\hline APTT & $x$ & $x$ & $x$ & $x$ & $x$ & $x$ \\
\hline $\mathrm{PT}+\mathrm{INR}$ & $x$ & & & & & \\
\hline Factor V & $x$ & & & & & \\
\hline Fibrinogen & $x$ & & & & & \\
\hline $\begin{array}{l}\text { Max of } 10 \mathrm{~mL} \\
\text { citrate plasma }\end{array}$ & $x$ & $x$ & $x$ & $x$ & $x$ & $x$ \\
\hline
\end{tabular}

Abbreviations: AF, alkaline phosphatase; ALAT, alanine aminotransaminase; APTT, activated partial thromboplastin time; ASAT, aspartate aminotransferase; EHL, extended half-life; FIX, factor IX; FV, factor V; FVIII, factor VIII; GGT, gamma-glutamyltransferase; INR, international normalized ratio; LDH, lactate dehydrogenase; PFA, platelet function assay; PK, pharmacokinetic; PT, prothrombin time; SHL, standard half-life; T, time point; VWF:Act, von Willebrand factor activity; VWF:Ag, von Willebrand factor antigen; VWF:CB, von Willebrand factor collagen binding; VWFpp, von Willebrand factor propeptide.

${ }^{\mathrm{a}}$ Only in case of an EHL concentrate.

together to explore the predictive performance of PK-guided dosing in real life.

\section{Statistical Analysis}

Continuous data will be expressed as mean and standard deviation when normally distributed or median and interquartile range when not normally distributed. Categorical data will be expressed as frequency and percentage.

As described in the primary study endpoint, the predictive performance of PK-guided dosing is deemed acceptable when at least $80 \%$ of the actual FVIII/FIX levels are within $\pm 25 \%$ of the predicted (target) values as stated by treating professional. Both the mean error between the predicted and observed factor level and the mean absolute difference of the predicted level will be calculated. No significant bias presented as zero is included in the $95 \%$ confidence interval (CI) of the mean error. Moreover, differences between the predictive performance of different factor concentrates and age groups will be investigated and described.

Association of factor levels with bleedings will be described according to sub classifications. Comparisons of the $A B R$ and joint status before and during PK-guidance will be 
Table 3 Laboratory tests during initial PK-guided treatment and follow-up

\begin{tabular}{|c|c|c|c|c|c|}
\hline & \multicolumn{3}{|c|}{$\begin{array}{l}\text { Initial PK-guided treatment } \\
\text { (12 weeks) }\end{array}$} & \multirow{2}{*}{$\begin{array}{l}\text { Follow-up } \\
\text { treatment } \\
\text { under PK } \\
\text { guidance } \\
\text { (24 weeks) } \\
1\end{array}$} & \multirow{2}{*}{$\begin{array}{l}\text { Bleeds (only when } \\
\text { blood sampling is } \\
\text { clinically indicated, } \\
\text { during total study } \\
\text { period) } \\
\text { additional }\end{array}$} \\
\hline Visit & 1 & 2 & 3 & & \\
\hline \multicolumn{6}{|l|}{ Hemophilia A } \\
\hline FVIII & $x$ & $x$ & $x$ & $x$ & $x$ \\
\hline FIX & & & & & $x$ \\
\hline VWF:Ag & & & & & $x$ \\
\hline VWF:Act & & & & & $x$ \\
\hline VWF:CB & & & & & $x$ \\
\hline VWFpp & & & & & $x$ \\
\hline APTT & $x$ & $x$ & $x$ & $x$ & $x$ \\
\hline Inhibitor FVIII & $x$ & & & $x$ & $(\mathrm{X})$ \\
\hline $\begin{array}{l}\text { Max of } 10-\mathrm{mL} \\
\text { citrate plasma }\end{array}$ & $x$ & $x$ & $x$ & $x$ & $x$ \\
\hline \multicolumn{6}{|l|}{ Hemophilia B } \\
\hline FIX & $x$ & $x$ & $x$ & $x$ & $x$ \\
\hline FVIII & & & & & $x$ \\
\hline VWF:Ag & & & & & $x$ \\
\hline VWF:Act & & & & & $x$ \\
\hline VWF:CB & & & & & $x$ \\
\hline VWFpp & & & & & $x$ \\
\hline APTT & $x$ & $x$ & $x$ & $x$ & $x$ \\
\hline Inhibitor FIX & & & $x$ & $x$ & $(X)$ \\
\hline $\begin{array}{l}\text { Max of } 10-\mathrm{mL} \\
\text { citrate plasma }\end{array}$ & $x$ & $x$ & $x$ & $x$ & $x$ \\
\hline
\end{tabular}

Abbreviations: APTT, activated partial thromboplastin time; FIX, factor IX; FVIII, factor VIII; PK, pharmacokinetic; VWF:Act, von Willebrand factor activity; VWF:Ag, von Willebrand factor antigen; VWF:CB, von Willebrand factor collagen binding; VWFpp, von Willebrand factor propeptide.

analyzed using a paired t-test or Wilcoxon's test, depending on the distribution.

$\mathrm{R}$ (version 4.0.3) will be used for statistical analysis.

\section{Ethical Considerations}

The study protocol was approved by the Medical Ethics Board of the Erasmus MC, University Medical Center Rotterdam, the Netherlands, and approved by all boards of all participating hospitals.

\section{Registration}

The trial is registered at the Dutch Trial register with trial number: NTR7523 (www.trialregister.nl).

\section{Conclusion}

The proposed study aims to investigate the reliability and feasibility of PK-guided prophylactic dosing of factor concentrates in hemophilia-A and -B patients in daily clinical practice. Moreover, the collected real-world data will lead to enrichment of current population PK models and an increased insight in the association of FVIII/FIX levels with bleeding episodes and daily activities.

Note

This study is part of the OPTI-CLOT Research Programme (Patient tailOred PharmacokineTIc-guided dosing of CLOTting factor concentrates and desmopressin in bleeding disorders)," an (inter)national multicenter study aiming to implement pharmacokinetic (PK)-guided dosing of replacement therapy and desmopressin by initiating studies to demonstrate its implications and feasibility, improve PK models, and to increase knowledge of patienttailored PK-guided dosing which is currently part of the SYMPHONY consortium that aims to unravel the origins of the interindividual variation in bleeding phenotype in order to install personalized treatment in all inherited bleeding disorders. 


\section{Funding}

This study was funded by Innovatiefonds Zorgverzekeraars, grant number: Project 3216; and Nederlandse Organisatie voor Wetenschappelijk Onderzoek, grant number: NWA.1160.18.038.

\section{Conflict of Interest}

M.H.C has received grants from governmental and societal research institutes, for example, NWO-ZonMW, NWONWA, Innovation fund, from private funds, institutional grants and unrestricted investigator research grants/educational, and travel funding from the following companies over the years: Pfizer, Baxter/Baxalta/Shire, Bayer Schering Pharma, CSL Behring, Sobi Biogen, Novo Nordisk, Novartis, Nordic Pharma and Roche, and has served as a member on steering boards of Roche and Bayer. All grants, awards and fees go to the Erasmus MC as an institution. R.A.A.M. has received governmental and societal research institutes such as NWO, ZonMW, and Innovation Fund and unrestricted investigator research grants from Baxter/Baxalta/Shire/Takeda, Bayer, CSL Behring, and Sobi. He has served as an advisor for Bayer, CSL Behring, Merck Sharp \& Dohme, and Baxter/Baxalta/Shire/Takeda. All grants and fees were paid to the institution.

H.J.C.J.E. received research support from CSL Behring outside the scope of this project and fees for educational activities from Roche and Celgene which fees go to the institution.

F.W.G.L. received unrestricted research grants from CSL Behring, Shire/Takeda, SOBI, and UniQure. He is a consultant for CSL Behring, Takeda, Biomarin and UniQure of which the fees go to the Erasmus MC as an institution. He served as DSMB member of a study sponsored by Roche. K.M. reports speaker fees from Bayer and Alexion, participation in trial steering committee for Bayer, consulting fees from UniQure, participation in data monitoring and endpoint adjudication committee for Octapharma. All fees are paid to the institution.

\section{Acknowledgments}

All authors would like to thank the OPTI-CLOT study group as a whole for their support and Iris van Moort and Jessica Heijdra for their involvement and participation.

The SYMPHONY consortium which aims to orchestrate personalized treatment in patients with bleeding disorders, is a unique collaboration between patients, health care professionals, and translational and fundamental researchers specialized in inherited bleeding disorders, as well as experts from multiple disciplines. It aims to identify the best treatment choice for each individual based on bleeding phenotype. To achieve this goal, workpackages (WP) have been organized according to three themes, for example, diagnostics (WPs 3\&4), treatment (WPs 5-9), and fundamental Research (WPs 10-12). This research received funding from the Netherlands Organization for Scientific Research (NWO) in the framework of the NWA-ORC Call grant agreement NWA.1160.18.038.
Principal investigator: M.H.C. Project manager: Dr. S.H. Reitsma.

Beneficiaries of the SYMPHONY consortium: Erasmus MC and Erasmus MC Sophia Children's Hospital, University Medical Center Rotterdam, project leadership and coordination; Sanquin Diagnostics; Sanquin Research; Amsterdam University Medical Centers; University Medical Center Groningen; University Medical Center Utrecht; Leiden University Medical Center; Radboud University Medical Center; Netherlands Society of Hemophilia Patients (NVHP); Netherlands Society for Thrombosis and Hemostasis (NVTH); and Bayer B.V., CSL Behring B. V., Swedish Orphan Biovitrum (Belgium) BVBA/SPRL.

\section{References}

1 Srivastava A, Brewer AK, Mauser-Bunschoten EP, et al; Treatment Guidelines Working Group on Behalf of The World Federation Of Hemophilia. Guidelines for the management of hemophilia. Haemophlia 2013;19(Suppl 1):e1-e47 PubMed

2 Leebeek FWG, Mauser-Bunschoten EP. Richtlijn diagnostiek en behandeling van hemofilie en aanverwante hemostase stoornissen. Utrecht: Van Zuiden Communications BV; 2009

3 Ahlberg A. Haemophilia in Sweden. VII. Incidence, treatment and prophylaxis of arthropathy and other musculo-skeletal manifestations of haemophilia A and B. Acta Orthop Scand Suppl 1965 (Suppl 7777:3-132

4 Björkman S, Oh M, Spotts G, et al. Population pharmacokinetics of recombinant factor VIII: the relationships of pharmacokinetics to age and body weight. Blood 2012;119(02):612-618

5 Björkman S. Prophylactic dosing of factor VIII and factor IX from a clinical pharmacokinetic perspective. Haemophilia 2003;9 (Suppl 1):101-108, discussion 109-110

6 Björkman S, Shapiro AD, Berntorp E. Pharmacokinetics of recombinant factor IX in relation to age of the patient: implications for dosing in prophylaxis. Haemophilia 2001;7(02):133-139

7 Chelle P, Yeung CHT, Croteau SE, et al. Development and validation of a population-pharmacokinetic model for Rurioctacog Alfa Pegol (Adynovate): a report on behalf of the WAPPS-Hemo Investigators Ad Hoc subgroup. Clin Pharmacokinet 2020;59 (02):245-256

8 Zhang Y, Roberts J, Tortorici M, et al. Population pharmacokinetics of recombinant coagulation factor VIII-SingleChain in patients with severe hemophilia A. J Thromb Haemost 2017;15(06): 1106-1114

9 Allard Q, Djerada Z, Pouplard C, et al. Real life population pharmacokinetics modelling of eight factors VIII in patients with severe haemophilia A: is it always relevant to switch to an extended half-life? Pharmaceutics 2020;12(04):E380

10 McEneny-King A, Chelle P, Foster G, Keepanasseril A, Iorio A, Edginton AN. Development and evaluation of a generic population pharmacokinetic model for standard half-life factor VIII for use in dose individualization. J Pharmacokinet Pharmacodyn 2019;46 (05):411-426

11 Brekkan A, Berntorp E, Jensen K, Nielsen EI, Jönsson S. Population pharmacokinetics of plasma-derived factor IX: procedures for dose individualization. J Thromb Haemost 2016;14(04):724-732

12 Diao L, Li S, Ludden T, Gobburu J, Nestorov I, Jiang H. Population pharmacokinetic modelling of recombinant factor IX Fc fusion protein (rFIXFc) in patients with haemophilia B. Clin Pharmacokinet 2014;53(05):467-477

13 Björkman S. Population pharmacokinetics of recombinant factor IX: implications for dose tailoring. Haemophilia 2013;19(05):753-757

14 Suzuki A, Tomono Y, Korth-Bradley JM. Population pharmacokinetic modelling of factor IX activity after administration of 
recombinant factor IX in patients with haemophilia B. Haemophilia 2016;22(05):e359-e366

15 Nestorov I, Neelakantan S, Ludden TM, Li S, Jiang H, Rogge M. Population pharmacokinetics of recombinant factor VIII Fc fusion protein. Clin Pharmacol Drug Dev 2015;4(03):163-174

16 Bukkems LH, Heijdra JM, Mathias M, et al; for UK-EHL Outcomes Registry OPTI-CLOT Collaboration. A novel, enriched population pharmacokinetic model for recombinant factor VIII-Fc fusion protein concentrate in hemophilia A patients. Thromb Haemost 2020;120(05):747-757

17 Shah A, Solms A, Wiegmann S, et al. Direct comparison of two extended-half-life recombinant FVIII products: a randomized, crossover pharmacokinetic study in patients with severe hemophilia A. Ann Hematol 2019;98(09):2035-2044

18 Zhang Y, Roberts J, Bensen-Kennedy D, et al. Population pharmacokinetics of a new long-acting recombinant coagulation factor IX albumin fusion protein for patients with severe hemophilia B. J Thromb Haemost 2016;14(11):2132-2140

19 Solms A, Iorio A, Ahsman MJ, et al. Favorable pharmacokinetic characteristics of extended-half-life recombinant factor VIII BAY 94-9027 enable robust individual profiling using a population pharmacokinetic approach. Clin Pharmacokinet 2020;59(05): 605-616

20 Solms A, Shah A, Berntorp E, et al. Direct comparison of two extended half-life PEGylated recombinant FVIII products: a randomized, crossover pharmacokinetic study in patients with severe hemophilia A. Ann Hematol 2020;99(11): 2689-2698

21 Björkman S, Folkesson A, Jönsson S. Pharmacokinetics and dose requirements of factor VIII over the age range 3-74 years: a population analysis based on 50 patients with long-term prophylactic treatment for haemophilia A. Eur J Clin Pharmacol 2009;65 (10):989-998

22 Stass $H$. Determination of minimal sampling time points for reliable pharmacokinetic evaluation of recombinant factor VIII an exploratory population pharmacokinetic analysis in paediatric patients suffering from severe haemophilia. Haemophilia 2006; 12:50-55

23 Garmann D, McLeay S, Shah A, Vis P, Maas Enriquez M, Ploeger BA. Population pharmacokinetic characterization of BAY 81-8973, a full-length recombinant factor VIII: lessons learned - importance of including samples with factor VIII levels below the quantitation limit. Haemophilia 2017;23(04):528-537

24 Shah A, Solms A, Garmann D, et al. Improved pharmacokinetics with BAY 81-8973 versus antihemophilic factor (recombinant) plasma/albumin-free method: a randomized pharmacokinetic study in patients with severe hemophilia A. Clin Pharmacokinet 2017;56(09):1045-1055

25 Björkman S, Ahlén V. Population pharmacokinetics of plasmaderived factor IX in adult patients with haemophilia B: implica- tions for dosing in prophylaxis. Eur J Clin Pharmacol 2012;68(06): 969-977

26 Bolon-Larger M, Chamouard V, Bressolle F, Boulieu R. A limited sampling strategy for estimating individual pharmacokinetic parameters of coagulation factor VIII in patients with hemophilia A. Ther Drug Monit 2007;29(01):20-26

27 Jiménez-Yuste V, Lejniece S, Klamroth R, et al. The pharmacokinetics of a B-domain truncated recombinant factor VIII, turocto$\operatorname{cog}$ alfa (NovoEight), in patients with hemophilia A. J Thromb Haemost 2015;13(03):370-379

28 Tiede A, Abdul Karim F, Jiménez-Yuste V, et al. Factor VIII activity and bleeding risk during prophylaxis for severe hemophilia $A$ : a population pharmacokinetic model. Haematologica 2021;106 (07):1902-1909

29 Delavenne X, Dargaud Y, Ollier E, Négrier C. Dose tailoring of human cell line-derived recombinant factor VIII simoctocog alfa: Using a limited sampling strategy in patients with severe haemophilia A. Br J Clin Pharmacol 2019;85(04):771-781

30 Karafoulidou A, Suarez E, Anastasopoulou I, et al. Population pharmacokinetics of recombinant factor VIII:C (ReFacto) in adult HIV-negative and HIV-positive haemophilia patients. Eur J Clin Pharmacol 2009;65(11):1121-1130

31 Collins PW, Møss J, Knobe K, Groth A, Colberg T, Watson E. Population pharmacokinetic modeling for dose setting of nonacog beta pegol (N9-GP), a glycoPEGylated recombinant factor IX. J Thromb Haemost 2012;10(11):2305-2312

32 Abrantes JA, Nielsen EI, Korth-Bradley J, Harnisch L, Jönsson S. Elucidation of factor VIII activity pharmacokinetics: a pooled population analysis in patients with hemophilia a treated with Moroctocog alfa. Clin Pharmacol Ther 2017;102(06):977-988

33 Hazendonk HC, Lock J, Mathôt RA, et al. Perioperative treatment of hemophilia A patients: blood group $O$ patients are at risk of bleeding complications. J Thromb Haemost 2016;14(03):468-478

34 Björkman S. Limited blood sampling for pharmacokinetic dose tailoring of FVIII in the prophylactic treatment of haemophilia A. Haemophilia 2010;16(04):597-605

35 Carlsson M, Berntorp E, Björkman S, Lethagen S, Ljung R. Improved cost-effectiveness by pharmacokinetic dosing of factor VIII in prophylactic treatment of haemophilia A. Haemophilia 1997;3(02):96-101

36 van Moort I, Preijers T, Bukkems LH, et al; OPTI-CLOT study group. Perioperative pharmacokinetic-guided factor VIII concentrate dosing in haemophilia (OPTI-CLOT trial): an open-label, multicentre, randomised, controlled trial. Lancet Haematol 2021;8 (07):e492-e502

37 Ragni MV, Croteau SE, Morfini M, Cnossen MH, Iorio ASubcommittee on Factor VIII, Factor IX, and Rare Bleeding Disorders. Pharmacokinetics and the transition to extended half-life factor concentrates: communication from the SSC of the ISTH.J Thromb Haemost 2018;16(07):1437-1441 